\title{
ON CERTAIN CONDITIONS FOR THE EXISTENCE OF INVARIANT LINEAR FUNCTIONALS
}

\author{
A. J. SCHWARTZ ${ }^{1}$
}

1. Introduction. The existence of an invariant measure is a hypothesis for many theorems in topological dynamics [2]. N. M. Krylov and N. N. Bogoliubov have demonstrated the existence of such a measure when a one-parameter transformation group acts on a compact metric space [1]. Their proof involves the sequential compactness of the space of normalized measures of a compact metric space.

In this paper we shall investigate the existence of invariant positive linear functionals which is equivalent to the existence of invariant Borel measures. A different and more general approach will be used from that of the above paper. We shall not assume the space being considered to be metrizable.

The author would like to express his gratitude to Professor Felix Haas for his encouragement during the writing of this paper. ${ }^{2}$

2. Notation. $M$ denotes a compact space and $\mathfrak{e}(M)$ the Banach space of real valued continuous functions over $M$ together with the sup norm

$$
\|\phi\|=\max \{|\phi(x)|: x \in M\} .
$$

$J(M)$ denotes the set of continuous functions mapping $M$ into itself. $\alpha$ is the zero function and $\pi$ the unit function of $\mathfrak{e}(M)$; i.e., $\alpha(M)=0$ and $\pi(M)=1$.

DEFINITION. If $S \subset J(M)$, by an $S$-functional we mean a normalized, positive, linear functional which is invariant under $\delta$; i.e., $L$ is an $\delta$-functional if $L$ is a linear functional and

$$
\begin{aligned}
& L(\pi)=1, \\
& \phi \geqq 0 \Rightarrow L(\phi) \geqq 0, \\
& g \in \mathcal{S} \text { and } \phi \in \mathfrak{C}(M) \Rightarrow L(\phi \circ g)=L(\phi) .
\end{aligned}
$$

Received by the editors August 8,1960 and, in revised form, November 14, 1960.

1 This work was supported by a National Science Foundation Cooperative Fellowship. It is part of the author's doctoral dissertation prepared under the direction of Professor Felix Haas at Wayne State University.

2 The author is indebted to the referee for calling his attention to the result of Mazur and Orlicz [3], noted below. 
Definition.

$$
D(M, s)=\left\{\phi=\sum_{i=1}^{n} \phi_{i} \circ g_{i}-\phi_{i}: \phi_{i} \in \mathcal{C}(M), g_{i} \in s\right\} .
$$

It is clear that $D(M, \S)$ is a linear subspace of $\mathfrak{C}(M)$.

3. A sufficient condition. We first prove the following:

THEOREM I. A sufficient condition for the existence of an S-functional is that

$$
\phi \in D(M, s) \Rightarrow \phi^{-1}(0) \neq \varnothing .
$$

Proof. Let $\mathcal{H C}$ be the subspace spanned by $D(M, \mathcal{S})$ and $\pi$. Then for each $\phi$ in $\mathcal{H}$, we have $\phi=\gamma+a \pi$ where $\gamma \in D(M, s)$. Since $\pi^{-1}(0)=\varnothing$, $\pi \notin D(M, \delta)$, the representation is unique. Thus for every $\phi \in \mathcal{F}$ we may define

$$
L^{\prime}(\phi)=a .
$$

It is clear that $L^{\prime}$ is a linear functional defined on $\mathcal{F}$ such that $L^{\prime}(D(M, \delta))=0$. Moreover if $\phi=\gamma+a \pi$ then $\|\phi\| \geqq|a|$ and in case $\gamma=\alpha,\|\phi\|=|a|$. Therefore we have

$$
\text { norm } L^{\prime}=1=L^{\prime}(\pi) \text {. }
$$

Now, by a well-known corollary of the Hahn-Banach theorem, we may extend $L^{\prime}$ to a linear functional, $L$, on $\mathfrak{e}(M)$ with norm 1 . $L$ must satisfy (2), for if $\phi \in \mathfrak{e}(M)$ and $\phi \geqq 0$, letting

$$
c=1 /(1+\|\phi\|)
$$

we have

$$
\alpha \leqq c \phi \leqq \pi
$$

and therefore

$$
\|\pi-c \phi\| \leqq\|\pi\|=1
$$

so that

$$
L(\pi-c \phi) \leqq 1
$$

Thus

$$
L(\phi)=(1 / c) L(c \phi)=(1 / c)[L(\pi)-L(\pi-c \phi)] \geqq 0 .
$$

Finally, since $L(D(M, \S))=L^{\prime}(D(M, s))=0, L$ is an $s$-functional and the theorem is proven.

${ }^{8}$ This theorem may also be deduced from the more general result 2.43 of [3]. 
We next show that under certain conditions, $D(M, s)$ satisfies the hypothesis of Theorem I.

THEOREM II. Let $S \subset J(M)$ form a semi-group with respect to composition. Furthermore, let $x \in M$ be such that $S x$ is contained in a single component of $M$ and such that for any $g, h \in S$,

$$
g(h(x))=h(g(x)) \text {. }
$$

Then it follows that $\phi \in D(M, s) \Rightarrow \phi^{-1}(0) \neq \varnothing$.

Proof. Assume the contrary. Then for some $\phi=\sum_{k=1}^{n} \phi_{k} g_{k}-\phi_{k}$, $0 € \phi(M)$. Without loss of generality, we may assume

$$
\phi(y) \geqq d>0
$$

for all $y \in M^{\prime}$, the component of $M$ containing $S x$. On the other hand, we may choose an integer $N$ sufficiently large so that

$$
2 n\left\|\phi_{k}\right\|<N d
$$

for all $k, 1 \leqq k \leqq n$. Now consider

$$
\sum=\sum_{j_{1}=1}^{N} \cdots \sum_{j_{n}=1}^{N} \phi\left(g_{1}^{j_{1}}\left(\cdots\left(g_{n}^{j_{n}}(x)\right) \cdots\right)\right) .
$$

It follows from the fact that $s x \subset M^{\prime}$ and (') that $\sum \geqq N^{n} d$. On the other hand, we have

$$
\begin{aligned}
& \sum=\sum_{j_{1}=1}^{N} \cdots \sum_{j_{n}=1}^{N} \sum_{k=1}^{n}\left[\phi_{k}\left(g_{k}\left(g_{1}^{j_{1}}\left(\cdots\left(g_{n}^{j_{n}}(x)\right) \cdots\right)\right)\right)\right.\left.-\phi_{k}\left(g_{1}^{j_{1}}\left(\cdots\left(g_{n}^{j_{n}}(x)\right) \cdots\right)\right)\right\rfloor \\
&\left.\left.=\sum_{k=1}^{n} \sum_{j_{m}=1 ; m \neq k}^{N} \sum_{j_{k=1}^{N}\left\lfloor\phi _ { k } \left(g _ { 1 } ^ { j _ { 1 } } \left(\cdots \left( g_{k}^{j_{k+1}}(\cdots)\right.\right.\right.\right.}\left(\cdots\left(g_{n}^{j_{n}}(x)\right) \cdots\right)\right) \cdots\right) \\
&\left.-\phi_{k}\left(g_{1}^{j_{1}}\left(\cdots\left(g_{n}^{j_{n}}(x)\right) \cdots\right)\right)\right]
\end{aligned}
$$

since for $g, h \in \mathcal{S}, g(h(x))=h(g(x))$ by hypothesis. Therefore

$$
\begin{aligned}
\sum & =\sum_{k=1}^{n} \sum_{j_{m}=1 ; m \neq k}^{N}\left\lfloor\phi_{k}\left(g_{1}^{j_{1}}\left(\cdots\left(g_{k}^{N+1}\left(\cdots\left(g_{n}^{j_{n}}(x)\right) \cdots\right)\right) \cdots\right)\right)\right. \\
& \leqq n N^{n-1} \max _{k}\left\|\phi_{k}\right\| \\
& <N^{n} d,
\end{aligned}
$$


according to ("). This contradiction proves the theorem.

We note that in general, the condition of Theorem I is not necessary. This can readily be seen if one considers the case where $M$ consists of a discrete space with two points and $\delta$ is the group of permutations.

4. Necessity of the condition. If we impose certain auxiliary restrictions, the condition of Theorem I becomes a necessary one as we now show.

Notation. By $M^{x}$ we denote the component of $x$ in $M$.

THEOREM III. If $S \subset J(M)$ has the property that $S x \subset M^{x}$, for every $x \in M$, then there exists an S-functional only if

$$
\phi \in \mathscr{D}(M, s) \Rightarrow \phi^{-1}(0) \neq \varnothing .
$$

Proof. Let $L$ be an $\delta$-functional on $\mathcal{C}(M)$ and suppose there exists $\phi \in D(M, s)$ such that $0 \notin \phi(M)$. Let

$$
\begin{aligned}
& M^{\prime}=\{x \in M: \phi(x)>0\}, \\
& M^{\prime \prime}=\{x \in M: \phi(x)<0\} .
\end{aligned}
$$

Since $\phi$ is continuous. $M^{\prime}$ and $M^{\prime \prime}$ are open. Since $0 \notin \phi(M)$, $M=M^{\prime} \cup M^{\prime \prime}$. We also have $M^{\prime} \cap M^{\prime \prime}=\varnothing$. Thus $M^{\prime}$ and $M^{\prime \prime}$ are closed. It follows, then, that $\mu^{\prime}$, the characteristic function of $M^{\prime}$, is continuous. We may assume without loss of generality that $L\left(\mu^{\prime}\right)$ $=m>0$. Furthermore, since $M^{\prime}$ is compact, we have $\phi(x) \geqq d>0$ for all $x \in M^{\prime}$.

Let $\phi^{\prime} \in \mathfrak{C}(M)$ be defined by

$$
\phi^{\prime}(x)=\left\{\begin{array}{c}
\phi(x) \\
0
\end{array}\right.
$$

for $x \in M^{\prime}$, for $x \in M^{\prime \prime}$.

The continuity of $\phi^{\prime}$ follows from the fact that it is continuous on the open and closed sets $M^{\prime}$ and $M^{\prime \prime}$ and is consistently defined on their (empty) intersection. Since $\delta x \subset M^{x}$ for all $x$, it can easily be shown that $\phi^{\prime} \in D(M, \S)$. But from the fact that $\phi^{\prime} \geqq d \mu^{\prime}$ it follows that $L\left(\phi^{\prime}\right) \geqq d m>0$. However $L(D(M, \delta))=0$ since $L$ is an $\delta$-functional. This contradiction proves the theorem.

5. Applications. The following corollaries are consequences of the above theorems.

COROLlARY. If $G$ is a transformation group acting on a compact space $M$, and either $G$ or $M$ is connected, then there exists a $G$-functional if and only if $\phi \in D(M, G) \Rightarrow \phi^{-1}(0) \neq \varnothing$. 
COROLlARy. If $G$ is an abelian transformation group acting on a compact space $M$ and either $G$ or $M$ is connected, then there exists $a G$ functional.

While it can be shown that for a single transformation, $h$, of a compact metric space there always exists an $h$-functional (by using a method similar to that of Krylov and Bogoliubov), we conclude this article by exhibiting a compact space and two homeomorphisms which do not admit a functional invariant under both of them.

Example. Let $M$ be the unit circle $|z|=1$ in the complex plane. Consider the following homeomorphisms:

$$
\begin{aligned}
g(\exp [i \pi x]) & = \begin{cases}\exp \left[i \pi x^{2}\right], & \text { for } 0 \leqq x \leqq 1, \\
\exp \left[i \pi\left(1+(x-1)^{1 / 2}\right)\right], & \text { for } 1 \leqq x \leqq 2,\end{cases} \\
h(\exp [i \pi(1 / 2+y)]) & = \begin{cases}\exp \left[i \pi\left(1 / 2+y^{2}\right)\right], & \text { for } 0 \leqq y \leqq 1, \\
\exp \left[i \pi\left(3 / 2+(y-1)^{1 / 2}\right)\right] & \text { for } 1 \leqq y \leqq 2,\end{cases}
\end{aligned}
$$

and let

$$
\begin{aligned}
\phi(\exp [i \pi x]) & = \begin{cases}x, & \text { for } 0 \leqq x \leqq 1, \\
2-x, & \text { for } 1 \leqq x \leqq 2,\end{cases} \\
\beta(\exp [i \pi(1 / 2+y)]) & = \begin{cases}y, & \text { for } 0 \leqq y \leqq 1, \\
2-y, & \text { for } 1 \leqq y \leqq 2,\end{cases}
\end{aligned}
$$

then it follows from a direct computation that $\phi(g(z))-\phi(z)+\beta(h(z))$ $-\beta(z)<0$ for all $z$. Thus since $M$ is connected, Theorem III implies there cannot exist a functional invariant with respect to both $g$ and $h$.

\section{REFERENCES}

1. N. M. Bogoliubov and N. N. Krylov, La theorie génerale de la mesure et son application à l'étude des systèmes dynamiques de la mécanique non linéaire, Ann. of Math. vol. 38 (1937) pp. 65-113.

2. V. V. Nemyckii and V. V. Stepanov, Qualitative theory of differential equations, Gostehizdat, Moscow-Leningrad, 1947.

3. S. Mazur and W. Orlicz, Sur les espaces métriques lineaires. II, Studia Math. vol. 13 (1953) pp. 137-169.

Wayne State University 